\title{
Modernización ganadera bovina en el sur de Chile: el rol del médico veterinario Alfredo Neumann, 0sorno, siglo XX
}

\author{
Modernização e pecuária bovina no sul do Chile: o papel do médico veterinário Alfredo \\ Neumann, 0sorno, século XX
}

Livestock bovine modernization in the Southern Chile: the role of the veterinarian Alfredo Neumann, 0sorno,20th-Century

Eduardo Gallardo Martínez ${ }^{*}$

\section{Resumen}

Este artículo estudia la figura del médico veterinario chileno-alemán Alfredo Neumann Kuschel, destacándolo como un actor fundamental en el proceso de modernización de la ganadería bovina en Osorno, sur de Chile, durante el siglo XX. Se relaciona a Neumann con conceptos como modernización y transnacionalidad, en un ejercicio de carácter prosopográfico.

Palabras clave: Ganadería. Modernización. Transnacionalidad.

\section{Presentación}

La actual provincia de Osorno es el centro de la ganadería bovina en Chile. Este reducido territorio situado en el sur del país es su primer productor cárneo y lácteo, siendo esta actividad motor económico y símbolo cultural de esta localidad llamada "tierra de la carne y patria de la leche". La consolidación de Osorno en la ganadería no es sólo a nivel nacional, sino que también global: El año 2006 la empresa privada abrió allí la planta de cárneos más grande del país,

\footnotetext{
Magíster en Ciencias Humanas. Doctorando en historia, Instituto de Estudios Latinoamericanos y Freie Universität, Berlin. E-mail: egallardo@zedat.fu-berlin.de

Recebido em 20/10/2015 - Aprovado em 15/12/2015 http://dx.doi.org/10.5335/hdtv.16n.1.6265
} 
apuntando a procesar productos destinados a la Unión Europea y Estados Unidos, mientras que la transnacional Nestlé inauguró el 2012 en Osorno lo que se señaló como la planta lechera de Nestlé más moderna del mundo, sumándose a lo anterior la reciente e inédita exportación de más de 7.000 hembras vivas desde Osorno hacia China para mejorar la ganadería del gigante asiático, gracias a la calidad sanitaria y genética del ganado austral. Los productores regionales que lideran este presente son generalmente chilenos descendientes de alemanes, los cuales tienen un protagonismo histórico en esta actividad. ${ }^{1}$

Este artículo explora los antecedentes de tal situación contemporánea, proponiendo que en la primera mitad del siglo XX se inició en Osorno un proceso de modernización ganadera protagonizado por su elite o grupo rector: los chilenos descendientes de alemanes o chileno-alemanes, identificándose como uno de sus promotores al médico veterinario Alfredo Neumann Kuschel, un personaje inexplorado por la historiografía, por lo que el examen de su trayectoria pretende contribuir al conocimiento sobre el pasado ganadero en el sur de Chile. ${ }^{2}$ El rol de Neumann se relaciona con conceptos teóricos como modernización y transnacionalidad, mientras que desde lo metodológico se le analiza como un sujeto inserto en un colectivo social mayor y ante su contexto, realizando un ejercicio de carácter prosopográfico. Para el examen de este personaje se consultaron diversas fuentes, entre otras, sus propias publicaciones, textos conmemorativos y materiales de la Escuela Superior de Agricultura de Osorno, hoy Institu- to Profesional Agrario Adolfo Matthei (en adelante, ESAO) y la Sociedad Agrícola y Ganadera de Osorno (en adelante, SAGO). Este artículo se enmarca en una investigación más amplia sobre las relaciones chileno-alemanas en Osorno y su expresión en la modernización ganadera bovina para este territorio entre 1917 y 1938.

\section{Alfredo Neumann Kuschel, una trayectoria destacada (1903-2000)}

Alfredo Neumann nació y creció en un contexto dominado por conceptos como progreso y modernización, en la transición de los siglos XIX al XX. El estatus de lo moderno, comprendido en aquella época como un ideal o meta basada en los supuestos de racionalidad para una sociedad industrializada, debía alcanzarse mediante un proceso permanente de modernización. En tal lógica, las naciones sudamericanas observaron a Europa y luego a Estados Unidos como modelo, llevando a cabo transformaciones en la organización estatal y actividades económicas, mientras que en lo sociocultural, especialmente en las urbes, penetraba el influjo de las potencias en lo que se ha denominado como "sociedad de masas" (SUBERCASEAUX, 1997; RINKE, 2002). Nacido en Osorno el año 1903 en una familia de origen alemán, Neumann en su juventud fue testigo del acelerado proceso de modernización chileno comprendido entre el fin de la Guerra del Pacífico y la Crisis de 1929, mientras que en la región de donde él provenía se había consolidado una élite chileno-alemana en lo político, económico y sociocultural, debido al éxito 
de la inmigración germana desde mediados del siglo XIX, ocupando este grupo las mejores tierras en la depresión intermedia de las actuales regiones de Los Ríos y Los Lagos (BLANCPAIN, 1974; YOUNG, 1974; OJEDA-EBERT, 1984).

Alfredo Neumann visto como sujeto socio-cultural expresa la fusión germano-chilena, característica que lo sitúa como actor privilegiado para los contactos chileno-alemanes del territorio austral durante el siglo XX, esto a pesar de que no ocupó cargo político-diplomático alguno. Personajes como Neumann se pueden comprender como promotores de relaciones transnacionales, entendidas éstas como contactos internacionales estimulados paralelamente a las relaciones interestatales de carácter oficial, contactos que merecen atención debido a sus múltiples manifestaciones e influencias (RINKE, 1996; RISSE, 1999; IRIYE; SAUNIER, 2009). Neumann recibió inicialmente educación en la escuela alemana de Osorno, un espacio social que cumplía un importante rol en la promoción de costumbres y valores vinculados al "Deutschtum" o germanidad, trasladándose luego a la zona central del país, un escenario de mayor influencia cultural chilena donde completó su formación secundaria y superior. Como otros descendientes de alemanes, Neumann era jurídicamente chileno y desarrolló su trayectoria personal y profesional en el país, sin embargo, debido a sus orígenes y el medio socio-cultural en el que se desenvolvió llevaba también consigo una fuerte influencia germana. Esta mezcla fue determinante en sus actividades, induciéndolo a él y sus semejantes hacia la resolución de sus pro- blemáticas observando no sólo las posibilidades del medio chileno, sino también las que ofrecían la tierra de sus ancestros al otro lado del Atlántico, convirtiéndose así en un actor transnacional para las relaciones chileno-alemanas.

El vínculo de Neumann con la comunidad chileno-alemana en el sur es extenso y considera múltiples aspectos. Entre ellos se cuenta su rol como uno de los iniciadores de la ESAO, fundada por chileno-alemanes en 1932, donde ejerció cargos docentes y directivos durante un largo periodo del siglo XX y en la que debido a sus gestiones recibió como donación un taller de maquinaria agrícola y materiales de estudio por parte del gobierno de Alemania Federal en los años 60, siendo aquella escuela desde sus orígenes un centro de transferencia de conocimiento de origen alemán hacia y desde el sur de Chile. Hombre con intereses multifacéticos, desarrolló además escritos donde destaca su orientación hacia lo germano, tal como lo ilustran sus publicaciones, "Alexander von Humboldt: Su obra y su influencia en América" y "Presencia de la mujer en la inmigración alemana en el sur de Chile". Debido a su destacado rol como promotor de los contactos chileno-alemanes, Neumann recibió en 1979 de parte de la Liga Chileno-Alemana de Santiago la Medalla Carlos Anwandter, mientras que en 1986 el gobierno de Alemania Federal lo condecoró con su máxima distinción civil, la Cruz al Mérito, a lo cual se suma su protagónica participación en diversas organizaciones, por ejemplo, Club Alemán de Osorno, Colegio Médico Veterinario, Rotary Club y masonería de Osorno, entre otras, todo lo cual le valió una serie de reconocimientos 
públicos, como lo fue ser designado en 1987 hijo ilustre de Osorno. ${ }^{3}$

Desde el punto de vista profesional, Neumann cuenta también con una destacada trayectoria. Estudió veterinaria en Santiago cuando esta profesión llevaba poco tiempo de desarrollo en el país (SIEVERS, 1971; BASULTO, 2015), titulándose como médico veterinario en 1927 y vinculándose luego al ejército en el trabajo con equinos en la zona norte, un hecho significativo por cuanto la ciencia veterinaria en Chile tuvo desde sus orígenes fuertes vínculos con el quehacer militar. Tras un breve paso por la zona central, a partir de 1930 se estableció en el sur integrando el servicio público como veterinario regional desde Valdivia hasta Chiloé y Aysén, donde ocupó su jefatura. En el resto de su desempeño profesional ejerció docencia en educación superior, sobre todo en la ESAO, participó como jurado oficial en exposiciones ganaderas regionales y viajó al extranjero en comisiones de gobierno y asistencia a congresos científicos, reiterando visitas a Estados Unidos, Latinoamérica y Europa, especialmente Alemania. Revisando las acciones y escritos de Neumann destaca su preocupación por la sanidad animal y la genética, ámbitos donde realizó estudios de campo y produjo considerable bibliografía. Sus trabajos fueron difundidos en el medio regional mediante la revista Agricultura Austral y la radioemisora de la SAGO. Debido a su trayectoria profesional, Neumann recibió múltiples reconocimientos, entre otros, la Orden al Mérito Gabriela Mistral otorgada por el gobierno chileno en 1982, Premio al Mejor Profesional de Agro de la Sociedad Nacional de Agricultura de Santiago en 1985 y premio Dr. Hugo Sievers Wicke, otorgado en 1987 por sus pares del Colegio Médico Veterinario de Chile (REPÚBLICA DE CHILE, 2001).

Figura 1 - Fotografía de Alfredo Neumann (de traje oscuro) junto al Presidente Carlos Ibáñez del Campo, ESAO, Osorno, 1954

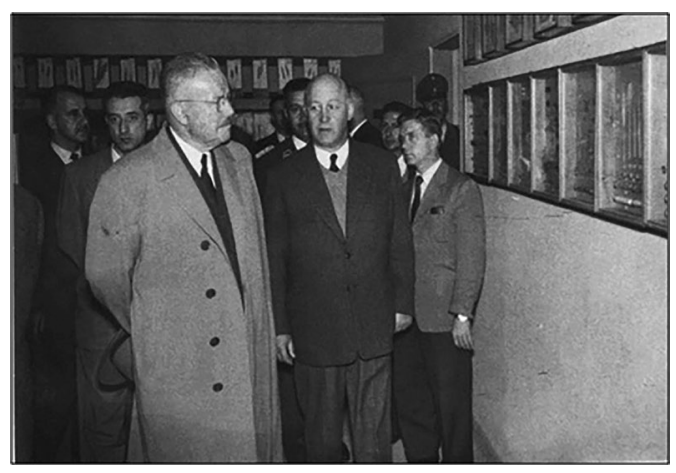

Fuente: FUNDACIÓN ADOLFO MATTHEI. Homenaje al Dr. Alfredo Neumann Kuschel. Osorno: Todográfica, 1994, p. 19.

\section{El rol modernizador de Alfredo Neumann en su contexto}

Alfredo Neumann se integró a su trabajo en el sur durante los años 30 en un complejo escenario agrario nacional y regional. Entre otros factores, los efectos de la Primera Guerra Mundial y la Crisis del 29 habían determinado para Chile el fin de su proceso de expansión agraria iniciado en la segunda mitad del siglo XIX (ROBLES, 2003), volcando en adelante su actividad hacia el contexto interno, en tanto el Estado miraba en dirección opuesta al agro manifestando interés creciente en industria y minería. Todo esto ocurría en un periodo de convulsión nacional determinado por un cambio constitucional en 1925, levantamientos militares y luchas político-sociales. Por otro lado, la 
literatura especializada de la época indica la existencia de un "problema agrario", resumido en la incapacidad del país para satisfacer sus necesidades alimentarias, llegando incluso a cuestionarse el régimen de propiedad (POBLETE, 1919; AGUIRRE CERDA, 1929; CORFO, 1940). Aún cargando con problemáticas económico-sociales irresueltas en cuanto a posesión de tierras, el sur se había integrado a la producción nacional sobre todo desde principios del siglo XX, destacado el territorio de la Araucanía a Chiloé en la ganadería bovina y cultivos de cereales y papa, mientras en Magallanes dominaban las estancias ovejeras. Los terratenientes del sur expresaron diferencias con sus pares de la zona central agrupados en la Sociedad Nacional de Agricultura, a la vez que se manifestaron insatisfechos con la política agraria estatal alegando que no protegía sus intereses, determinando aquello en la primera mitad del siglo XX la aparición de sociedades agrarias regionales y la creación en 1931 del Partido Agrario (ALMONACID, 2009). En Osorno se fundó en 1917 la SAGO con participación significativa de chilenos descendientes de alemanes, adquiriendo protagonismo en la relación de los agricultores del sur con el poder central (CARREÑO, 2008).

A pesar de este escenario adverso, hacia los años 30 Osorno se perfilaba como protagonista en la ganadería bovina de Chile. Desde antiguo este territorio se había vinculado a la explotación de vacunos y a partir de mediados del siglo XIX la inmigración alemana potenció tal actividad a través de la elaboración de charqui o carne salada y utilización del cuero, todo en el contexto de una pequeña y multifacética industria regional limitada en su productividad por la mala calidad de caminos y puentes, conformándose así un mercado lacustre y fluvial con centro en la ciudad y puerto industrial de Valdivia, situada más al norte, en un fenómeno fabril que decayó hacia 1914 a causa de factores internos y externos. La conexión definitiva vía ferrocarril de Osorno con el centro del país desde 1907 determinó su reorientación económica hacia la producción primaria, masificándose la crianza de vacunos para ser enviados vivos hacia la zona central del país, pasando de una existencia cercana a las 10.000 cabezas en 1891 a más 100.000 hacia 1910 y reflejándose la intensificación de esta actividad en la multiplicación de ferias de comercio ganadero en la zona, entre las que destacó la Feria de Osorno, propiedad del chileno-alemán Julio Buschmann (PERALTA, 1991; CAMUS, 1997). La literatura especializada de la época señalaba el potencial ganadero de este territorio, aunque indicaba introducir técnicas (CHILE, 1929; MATTHEI, 1939), mientras que los censos agropecuarios de $1930 \mathrm{y}$ 1936 indicaban al antiguo Departamento de Osorno en el primer lugar en cantidad de bovinos del país. ${ }^{4}$

El rol de Alfredo Neumann en la modernización ganadera bovina desde Osorno es amplio y se puede resumir en cuatro ámbitos, situándose en primer lugar su actividad como médico veterinario regional en el servicio público entre 1930 y 1957. Neumann se ocupó de un extenso territorio que abarcaba desde las actuales provincias de Valdivia hasta Chiloé y Aysén, tal como lo indica en su propio testimonio, " $\mathrm{mi}$ zona de trabajo era 
bastante grande. Y era uno de los pocos veterinarios que había en ese tiempo" (REVISTA VISUR, 1994, p. 6). Entre sus funciones estuvieron la inspección de carnes en mataderos municipales y asesorar en temas sanitarios a los ganaderos, mientras que su mayor dificultad fue la escasez de medios humanos y materiales. En una carta de la SAGO dirigida al Ministerio de Agricultura y publicada en 1935 por el diario local, se valoraba la acción de Neumann y se indicaba que por la importancia ganadera de Osorno se necesitaba allí mayor infraestructura veterinaria,

Por la razones expuestas, nos permitimos solicitar a V. S. se sirva arbitrar los medios para conseguir un mejoramiento de los servicios de veterinaria en el centro ganadero que forma Osorno, poniendo a disposición del señor Veterinario Regional don Alfredo Neumann los elementos necesarios para desarrollar más eficazmente su labor. Ello podría mientras tanto referirse como necesidades de carácter inmediato la instalación del Laboratorio de Diagnóstico de enfermedades del ganado (OSORNO..., 1935, p. 9).

La carta solicitaba además el aumento de sueldo de Neumann y proporcionarle ayudantes y facilidades para movilizarse. Tal aspecto en la trayectoria de este personaje es relevante, por cuanto orienta a reconocer las problemáticas del proceso de modernización ganadera expresadas en un irregular servicio fiscal durante la primera mitad del siglo $X X$, varias décadas antes que se crearan entidades estatales como los actuales Instituto de Investigaciones Agropecuarias y el Servicio Agrícola y Ganadero, fundados en 1964 y 1967, respectivamente.

Otro aspecto relevante en el servicio público de Neumann y que toca problemá- ticas de política agropecuaria para su época fue su experiencia en proyectos específicos. $\mathrm{Al}$ veterinario le correspondió intervenir en trabajos de campo para erradicar la brucelosis y fiebre aftosa, colaborando con destacados colegas:

En 1940 la Dirección General de Agricultura le encarga la confección de un proyecto para la erradicación de la Brucelosis en la zona, que fue aplicada íntegramente con positivos resultados. Al iniciarse la preparación de la vacuna anti-aftosa tipo Waldmann en Chile, colaboró activamente junto al Dr. Ramón Rodríguez y el profesor Dr. Waldmann en su aplicación, experimentación y estudio acompañando y secundando a éste durante su permanencia en el sur de Chile (FUNDACIÓN ADOLFO MATTHEI, 1994, p. 18).

El esfuerzo sanitario estatal personificado en Neumann, sin embargo, contrastaba con la internación de ganado argentino autorizada por el gobierno chileno. Los ganaderos del sur rechazaron esta situación argumentando razones económicas y sobre todo sanitarias, todo aquello ante un contexto regional donde conforme avanzaba el siglo XX se consolidaban fronteras e intereses nacionales, considerándose a la ganadería argentina como competencia e incluso perjuicio, de acuerdo con lo indicado por la SAGO,

La actual aftosa ha hecho su aparición junto con iniciarse la importación de ganado de la República Argentina, y se ha propagado con tal virulencia, que hace temer con fundadas razones, que sus daños sobrepasarán a la aftosa que azotó a la ganadería del país en el año de 1936... Por este motivo, consideramos que debería tomarse como medida de carácter inmediato, el cierre de la cordillera para los animales provenientes de esa república (AGRICULTURA AUSTRAL, 1939, p. 1). ${ }^{5}$ 
Estos reclamos hacia el Estado desde el grupo rector regional, sin embargo, están prácticamente ausentes en la literatura elaborada por Neumann, lo cual es comprensible dado su rol como funcionario público durante parte de su trayectoria.

El segundo ámbito de modernización ganadera donde intervino Neumann fue en la educación pecuaria, la cual se materializó en su acción llevada a cabo sobre todo en la ESAO. Debido a contactos profesionales y personales previos, este veterinario coincidió a principios de los años 30 en Osorno con el destacado ingeniero agrónomo chileno-alemán Adolfo Matthei, quien le planteó la idea de crear una escuela agronómica por iniciativa particular, tal como lo indica Neumann en su testimonio:

Luego de una serie de conversaciones insinuó la posibilidad de crear una Escuela Agrícola, que hacía mucha falta. Había algunas escuelitas agrícolas insuficientes, de una enseñanza muy inferior; no como para que fueran agricultores de alguna connotación. Hacía falta entonces, una institución que recibiera a los hijos de los dueños de fundo (REVISTA VISUR, 1994, p. 6).

La ESAO comenzó sus actividades lectivas en 1933 y Neumann figuró entre los primeros profesores, todos chilenos descendientes de alemanes, incluyendo además dos profesionales contratados desde Alemania, el Técnico Genético experto en vegetales Kurt Rehl y el Dr. en Agronomía Heinz Rödenbeek, mientras que el primer grupo de estudiantes eran en su totalidad chileno-alemanes. Las clases se impartían generalmente en idioma alemán y la formación era intensiva, totalizando dos años, similar a lo que en Europa se denominaba como "escuelas de invierno". Dadas algunas características de la ESAO, se advierte que en sus comienzos fue un proyecto concebido desde y para el grupo rector regional, destinado a satisfacer sus necesidades en formación agronómica (MATTHEI, 1933; GALLARDO, 2012).

La labor de Neumann en la ESAO fue bastante extensa, dedicada primero a la docencia y luego su dirección entre 1952 y 1994, totalizando entre ambas funciones más de 6 décadas. En materia veterinaria y considerando su rol en la escuela sobre todo durante la primera mitad del siglo XX, Neumann se puede considerar como protagonista en la génesis de una sistematización científica sobre el conocimiento animal para el sur de Chile, trabajando siempre inmerso en un espacio pedagógico de raíz germana, pues en lo teórico mucha de la literatura utilizada en los comienzos de la escuela estaba en idioma alemán, mientras que en lo práctico se realizaban trabajos de campo en fundos de chileno-alemanes que criaban bovinos finos provenientes de Alemania. La mejor evidencia de la labor pedagógica de Neumann es un sinnúmero de tesis de titulación de "peritos agrícolas" (título otorgado por la ESAO), material referido a una serie de prácticas pecuarias que no siempre obedecieron a un modelo rígido de emisión-recepción, dadas las características naturales particulares del sur chileno en comparación con el medio alemán. Por otro lado, cabe destacar que durante estos primeros años en la ESAO Neumann compartió con colegas alemanes, quienes a través de medios especializados e incluso informes dirigidos hacia autoridades alemanas dieron a conocer sus actividades en Chile, manifestándose así una red de transferencia 
de saber pecuario entre el sur de Chile y Alemania. En resumen, el rol de Neumann en la ESAO se estima en el contexto de un proceso de modernización agropecuaria protagonizado por actores transnacionales, el cual tenía como modelo a Alemania. ${ }^{6}$

El tercer ámbito de acción de Alfredo Neumann en la modernización ganadera austral se reconoce en las exposiciones pecuarias y la importación de bovinos finos desde Europa, destacando en los eventos anuales organizados por la SAGO. Desde 1917 y debido a la iniciativa de esta institución se realizó en Osorno una exposición agroganadera e industrial que rápidamente adquirió importancia para el sur del país, oportunidad en que se exhibían variados productos regionales, nacionales e importados y donde el ganado vacuno era protagonista, cabiendo destacar aquel acontecimiento como un espacio de relaciones transnacionales chileno-alemanas, debido al medio sociocultural donde se realizaba y la presencia de todo un aparato comercial de origen germano. La exposición era además un encuentro de carácter político, por cuanto en varias ocasiones asistió el Presidente de la República y altos funcionarios estatales, discutiéndose allí con el grupo rector regional diversos temas de interés. Tal evento se ha realizado de forma regular hasta hoy en Osorno.

Neumann en esta exposición ejerció reiteradas veces como jurado oficial del concurso de ganado fino, compuesto por ejemplares importados de Europa y su descendencia en tierras del sur, influyendo así con su criterio en los pasos técnicos a seguir por los criadores. Convencido por la lógica de la modernización aplicada a lo pecuario, Neu- mann valoraba la importación de hembras y sementales finos en una época en que aún no existían sistemas de inseminación artificial, sin embargo, también le preocupaba organizar la crianza basada en la selección genética mediante un registro regional de este ganado. En una conferencia durante la exposición de 1936, Neumann indicaba la conveniencia de tales registros debido a que racionalizaban la crianza y porque los vacunos inscritos aumentaban su valor. Hacia 1922 en Osorno se había abierto un registro genealógico, pero según Neumann éste debía actualizarse, razón por la cual la SAGO había formado una comisión para elaborar uno nuevo,

Este reglamento, encuadrado dentro de los principios científicos de la zootecnia moderna y de acuerdo con las reglamentaciones extranjeras hasta donde nuestras modalidades, condiciones de explotación y métodos lo permiten, asegura una documentación exacta, propende al fomento ganadero y estimula la actividad particular (NEUMANN, 1936b, p. 542).

De acuerdo con Neumann, la modernización ganadera austral, si bien inspirada en modelos foráneos, no debía seguir un patrón rígido de emisión-recepción, adecuándose así al medio local. En suma, se percibe que a través de este tipo de prácticas se fue configurando la especialización hacia la producción lechera y cárnea que hoy caracteriza a Osorno.

Por último, el cuarto ámbito de modernización ganadera en el que destacó Alfredo Neumann fue la literatura técnica y difusión radial de temas pecuarios, comprendiendo aquel la promoción del conocimiento veterinario mediante las herramientas de la 
modernidad. En 1932 apareció su primera publicación, "Tratamiento de las enfermedades del ganado", prologada por el ganadero Julio Buschmann, presidente de la SAGO, quien señalaba,

La obra que presenta el Dr. Neumann contiene una descripción sistemática completa, de fácil comprensión en que se enumeran las enfermedades más comunes del ganado y de las que con más frecuencia afectan a la ganadería del sur del país", agregando luego que, "Es por esto que en las bibliotecas de nuestros ganaderos y agricultores no debe faltar a fin de que puedan recurrir a ella con frecuencia (NEUMANN, 1932, p. 1).

Diez años más tarde Neumann publicó "Tres problemas que afectan a la producción ganadera", el cual recoge parte de sus conferencias radiales ofrecidas a través de la radioemisora de la SAGO, creada en 1939 como una novedad tecnológica para Osorno, mientras que en 1942 publicó "Enfermedades del ganado", una reedición ampliada de su trabajo de 1932, donde Neumann abarca la sanidad animal a nivel de país, incluyendo al final un resumen de criaderos. El libro fue prologado por el criador chileno-alemán Bernardo Minte, quien celebró el texto señalando, "aparte del aumento en el formato, de un mayor número de páginas y de su modernización, va profusamente ilustrada lo que la hace más interesante y facilita la comprensión" (NEUMANN, 1946, p. V). Posteriormente Neumann publicó un texto conmemorativo pero con interesante descripción acerca de Bernardo Minte, pionero en la importación de bovinos finos, donde informa sobre los orígenes de esta práctica en el sur de Chile, para terminar cerrando su producción bibliográfica en los años 90 con un trabajo sobre el Pudú, un ciervo patagónico en extinción que Neumann crió en cautiverio en un zoológico regional de especies nativas creado por su iniciativa en la ESAO.?

Aparte de los libros, el medio que Neumann utilizó con regularidad para difundir sus trabajos fue la revista institucional de la SAGO, Agricultura Austral. Allí se le puede observar como un autor estrictamente técnico, pues generalmente se abstuvo de abordar temas de política agropecuaria y menos discutir el acontecer del convulsionado escenario político nacional e internacional de la primera mitad del siglo XX. En este sentido, Neumann se diferencia de otros autores provenientes del grupo chileno-alemán en Osorno que si abordaron temas políticos, por ejemplo, Álvaro Bórquez Scheuch y sobre todo Adolfo Matthei (BÓRQUEZ, 1929; MATTHEI, 1939). Neumann además de sus propios escritos para Agricultura Austral tradujo para esta revista contenidos de medios alemanes como "Deutsche Landwirtschaftliche Presse" (NEUMANN, 1936a), por lo que se estima a esta publicación regional como difusora de conocimiento pecuario de origen germano. Lo anterior tiene más validez si se consideran también para esta revista las intervenciones de alemanes como Kurt Rehl y Heinz Rödenbeek, docentes de la ESAO, además de diversos artículos sobre el quehacer agropecuario de países considerados modernos, entre ellos, Alemania. El prestigio que alcanzó Agricultura Austral motivó la participación en sus contenidos por parte de destacados veterinarios chilenos, entre otros, Hugo Sievers, Arístides Ramírez, René Soriano y Germán Greve Silva. Este último elogió el trabajo pecuario en Osorno, 
Muchas veces hemos expresado que la ganadería sureña, tal cual se presenta en Osorno, es la lección objetiva más ponderable de las actividades que se desarrollan en esas provincias para el fomento de la producción agrícola. Esos ganados presentan la obra de muchos emprendedores e infatigables hombres de trabajo (GREVE SILVA, 1938, p. 1172).

La intervención de Neumann y otros especialistas en esta publicación regional permite aproximarse con más detalle a la modernización ganadera, siendo además esta revista una fuente recomendable para examinar la trayectoria silvoagropecuaria en el cono sur americano.

\section{Conclusiones}

Luego de lo expuesto, se interpreta que a través de la experiencia de Alfredo Neumann es posible reconocer una serie de acciones de modernización pecuaria iniciadas en la primera mitad del siglo XX en Osorno, originándose allí el protagonismo de este territorio en la ganadería bovina de Chile. Durante la segunda mitad de esta centuria tal protagonismo tendió a consolidarse con la instalación de infraestructura industrial, materializada en hitos como la creación del matadero modelo en 1938, la planta lechera de Nestlé en 1945 y el frigorífico de Osorno en 1957, influyendo todo aquello en la autonomía productiva de este territorio respecto a la zona central, más su progresiva especialización hacia los rubros cárneo y lácteo. Por otro lado, en la segunda mitad del siglo XX el gremio agro-ganadero se vio fortalecido con la presencia de una serie de cooperativas lecheras australes, por ejemplo, la Coo- perativa Agrícola y Lechera de Osorno y la Cooperativa Agrícola y Lechera de la Unión (KELLER, 1956; ROMÁN; VILLALOBOS, 1958; SANTANA, 2006). Este panorama resulta difícil de comprender si no se toman en cuenta los fenómenos de transnacionalidad y modernización protagonizados por actores como Alfredo Neumann.

En suma, este médico veterinario chileno-alemán se estima como un sujeto que personifica durante el siglo XX en el sur de Chile la modernidad y la transnacionalidad. Desde su quehacer profesional asumió los principios del proceso de modernización y concurrió a ejecutarlos en el escenario del sur de Chile, manifestando predilección por el modelo alemán. Dada su condición socio-cultural mixta, Neumann promovió desde Osorno una serie de contactos chileno-alemanes de carácter transnacional, los cuales tienen repercusión hasta la actualidad. En su trabajo específico como médico veterinario, la genética y la sanidad animal fueron aspectos clave del manejo pecuario que aquel incentivó, coincidiendo con las características que hoy permiten a la ganadería bovina de Osorno tener protagonismo en la era global. Al observar otros aspectos del contexto donde surge y se desarrolla este proceso de modernización durante el siglo XX, surgen, sin embargo, más cuestiones que apuntan a descifrar las contradicciones de este tipo de procesos, al no advertir una inclusión homogénea de todos los actores sociales que conforman este territorio austral, por ejemplo, habitantes de origen chileno e indígena, pudiendo interpretarse que tal proceso ha estado monopolizado y orientado preferentemente hacia la elite o grupo rector regional. 


\section{Resumo}

Este artigo estuda a figura do médico veterinário chileno-alemão Alfredo Neumann Kuschel, destacando-o como um ator fundamental no processo de modernização da pecuária bovina em Osorno, sul do Chile, durante o século XX. Relacionam-se a Neumann conceitos como modernização e transnacionalidade, em um exercício de caráter prosopográfico.

Palavras-chave: Pecuária. Modernização. Transnacionalidade.

\section{Abstract}

This paper addresses the leading figure of the Chilean-German veterinarian Alfredo Neumann Kuschel, highlighting him as fundamental actor in the Livestock Bovine modernization process at southern Chile during the 20th-Century. Neumann is connected with concepts such as modernisation and transnationality in a prosopographical exercise.

Keywords: Livestock. Modernisation. Transnationality.

\section{Notas}

1 El último censo agropecuario (2007), indica a la Provincia de Osorno en el primer lugar nacional en cantidad de ganado bovino, con 531.845 cabezas de un total de 3.789.697 cabezas. Disponible en: < http:/www.ine.cl/canales/chile_estadistico/ estadisticas_agropecuarias/estadisticas_pecuarias/ pdf/completabovinos09.pdf $>$. En la producción lechera de Osorno, la estadística reciente de la Federación Nacional de Productores de Leche señala al conjunto de plantas industriales de Osorno en el primer lugar nacional en recepción de leche fresca, con un total aproximado para el periodo enero-mayo 2015 de 251 millones de litros, en un total nacional aproximado de 800 millones de litros. Disponible en: < http://www.fedeleche.cl/estd/PDF/ recepcion.pdf $>$. Para la planta faenadora en Osorno, ver: < http://www.lanacion.cl/noticias/regiones/ inauguran-planta-faenadora-de-bovinos-en-osorno/2006-12-18/163309.html>. Para la inauguración de la planta de Nestlé en Osorno, ver: < http:// www.portalechero.com/innovaportal/v/2291/1/ innova.front/nestle_inaugura_en_osorno_su_planta_mas_moderna_en_el_mundo.html $>$. Para la exportación de ganado desde Osorno hacia China, ver: <http://www.fedeleche.cl/site/index.php/ noticias1/865-fegosa-se-asocia-a-firma-brasilena-para-enviar-7-300-vaquillas-a-china $>$.

2 Para trabajos sobre ganadería en el sur de Chile, ver, entre otros: PINTO, Jorge. Ganadería y empresarios ganaderos en la Araucanía. Historia, Santiago, v. 2, n. 44, p. 369-400, 2011; MARTINIC, Mateo. Recordando a un imperio pastoril: la sociedad explotadora de Tierra del Fuego (1893-1973). Magallania, Punta Arenas, v. 39, n. 1, p. 5-32, 2011; CARREÑO, Luis. Ganado argentino y curtiembres de Valdivia, 1850-1900. En: PINTO, Jorge. Araucanía siglos $X I X$ y $X X$ Economía migraciones y marginalidad. Osorno: Universidad de Los Lagos, 2011; MÉNDEZ, Laura; MUÑOZ, Jorge. Economías cordilleranas e intereses nacionales: genealogía de una relación. El caso de la Compañía Comercial y Ganadera Chile-Argentina (1895-1920). En: NUÑEZ, Rafael et al. (Ed.). Fronteras en movimiento e imaginarios geográficos. Santiago: RIL, 2013. p. 163-187.

3 Para las fuentes que informan sobre Alfredo Neumann en este artículo, ver: COREDE rindió homenaje al Dr. Alfredo Neumann. Diario Austral de Osorno, 25 marzo 1993; LA VIDA por un instituto. Entrevista a Alfredo Neumann. Visur, n. 1, p. 4-7, 1994; HOMENAJE al Dr. Alfredo Neumann Kuschel. Osorno: [s. n.], 1994; República Der Chile (2001, p. 30-37).

4 Sobre la cantidad de ganado bovino en Osorno, según los censos agropecuarios de 1930 y 1936, el Departamento de Osorno ocupa el primer lugar nacional en existencia de vacunos. En el primer censo contabiliza 183.798 bovinos $(7,6 \%)$ en un total nacional de 2.387. 940 y el segundo censo contabiliza $190.930(7,7 \%)$ en un total de 2.572.987. Cifras calculadas en base a: Dirección General de Estadística, Chile: Censo agropecuario 1929-1930. Santiago: Universo, 1933; del mismo autor: Agricultura 1935-36. Censo. Santiago: Universo, 1938.

El tráfico transcordillerano de ganado en el territorio de Osorno es de antigua data, habiendo motivado incluso la creación de sociedades binacionales por iniciativa particular, aunque estas organizaciones fueron de poca duración y sucumbieron conforme avanzó el siglo XX, debido a factores internos y externos, entre otros, la consolidación de las soberanías nacionales en estos territorios y los efectos de la primera guerra mundial, de acuerdo con estudios 
recientes. Para trabajos sobre estas sociedades, ver, entre otros: BLANCO, Graciela. Las sociedades anónimas cruzan los Andes: los inversores chilenos en Neuquén al comenzar el siglo XX. América Latina en la Historia Económica, México, v. 19, n. 2, p. 107-130, 2012.; MÉNDEZ, Laura; MUÑOZ, Jorge. Economías cordilleranas e intereses nacionales: Genealogía de una relación. El caso de la Compañía Comercial y Ganadera Chile-Argentina (1895-1920). En: NUÑEZ, Rafael et al. (Ed.). Fronteras en movimiento e imaginarios geográficos. Santiago: [s. n.], 2013. p. 163-187.

6 En las memorias de titulación de los peritos agrícolas de la ESAO hay mención a reconocidos especialistas alemanes de la primera mitad del siglo XX, entre otros, Ernst Klapp, Wilhelm Zorn, Oscar Kellner y Friedrich Aereboe, además de publicaciones como Deutsche Landwirtschaftliche Tierzucht y Deutsche Landwirtschaftliche Presse. Para trabajos de los profesores alemanes de la ESAO, ver, entre otros: RÖDENBEEK, Heinz. Der neue Kurs der Landwirtschaft in Chile. Mitteilungen der höheren Landwirtschaftlichen Schule zu Osorno (Chile), Osorno, n. 1, 1933; del mismo autor: Deutsche Züchterarbeit in Südamerika. Das Schwarzbunte Niederungsrind in SüdChile, insbesondere die hochzuchtwirtschaft Los Riscos. Deutsche Landwirtschaft Tierzucht, Jahrg. 39, n. 23, p. 357-361, 1935; Jahrg. 39, n. 24, p. 379-384, 1935; RÖDENBEEK, Heinz. Investigación científica sobre la crianza del "Bovino Overo Negro" en Chile, especialmente en el criadero "Los Riscos". Agricultura Austral, Osorno, a. V, n. 50, p. 644-661, 1937; REHL, Kurt. Grünland in Südchile. Osorno: Imprenta Central, 1938; del mismo autor: Chile austral, región excelente para cultivo de pasto. Agricultura Austral, a. VI, n. 68, p. 1588-1594, 1938. Para un informe de Rödenbeek dirigido a autoridades alemanas en Chile, ver: Die Einfuhr von deutschen Zuchtvieh in Chile. Osorno, 08.05.1934. En: Archivo Político del Ministerio de Relaciones Exteriores de Alemania, carpeta R245917. Para el tema de transferencias culturales y conocimientos, ver, entre otros: CELESTINI, Federico; MITTERBAUER, Helga (Ed.). Ver-rückte Kulturen: zur Dynamik Kultureller Transfers. Tübingen: Stauffenburg, 2003; DAHLMANN, Dittmar; REITH, Reinhold (Ed.). Elitenwanderung und Wissenstransfer im 19. und 20. Jahrhundert. Essen: Klartext, 2008; RODRIGUES, Helenice. Transferência de saberes: modalidades e possibilidades. História: Questões \& Debates, Curitiba, n. 53, p. 203-225, 2010.

7 Para estos dos últimos trabajos del médico veterinario, ver: NEUMANN, Alfredo. Don Bernardo Minte Sunkel, homenaje a su memoria. Osorno: [s. n.], 1975; del mismo autor: El Pudú. Contribución a su estudio etológico. Osorno: Impresur, 1992.

\section{Referencias}

AGRICULTURA AUSTRAL. Situación de la ganadería austral. Revista de la SAGO, Osorno, a. VI, n. 72, p. 1, 1939.

AGUIRRE CERDA, Pedro. El problema agrario. París: [s. n.], 1929.

ALMONACID, Fabián. La agricultura chilena discriminada (1910-1960): una mirada de las políticas estatales y el desarrollo sectorial desde el sur. Madrid: Consejo Superior de Investigaciones Científicas, 2009.

BASULTO, Sergio et al. Raíces de la medicina veterinaria en Chile. Santiago: Loyca Comunicación, 2015.

BLANCPAIN, Jean-Pierre. Les Allemands au Chili (1816-1945). Köln: Böhlau, 1974.

BÓRQUEZ, Álvaro. El problema de la inmigración. Osorno: La Prensa, 1929.

CAMUS, Pablo. Ordenamiento territorial y ferrocarril del sur en Osorno y Llanquihue: 1860-1960. Revista de Geografía del Norte Grande, Macul, n. 24, p. 165-173, 1997.

CARREÑO, Luis. La Sociedad Agrícola y Ganadera de Osorno (SAGO) y su aporte al desarrollo de la comunidad regional. Espacio Regional Revista de Estudios Sociales, Osorno, v. 5, n. 1, p. 35-48, 2008.

CHILE. Congreso Nacional. Homenaje en memoria de don Alfredo Neumann Kuschel. Diario de Sesiones de la Cámara de Diputados, Sesión 49 ${ }^{\circ}, 4$ abril 2001.p.30-37. Disponibleen:<http:/ / www.camara.cl/pdf.aspx?prmID $=4819 \% 20$ $\&$ prmTIPO=TEXTOSESION $>$. Acceso en: 15 mayo 2015.

Ministerio de Fomento. Estudio agronómico del Departamento de Osorno. Santiago: R. Harris, 1929.

CORFO. CORPORACIÓN DE FOMENTO DE LA PRODUCCIÓN. Plan de acción inmediata para la agricultura y explotaciones afines. Santiago: Letelier, 1940. 
FUNDACIÓN ADOLFO MATTHEI. Homenaje al Dr. Alfredo Neumann Kuschel. Osorno: Todográfica, 1994.

GALLARDO, Eduardo. El ingeniero agrónomo Dr. Adolfo Matthei y su rol en el desarrollo de la agricultura en Chile y Osorno. Osorno: Editorial Universidad de Los Lagos, 2012.

GREVE SILVA, Germán. Osorno en su carácter de emporio zootécnico. Agricultura Austral, Osorno, a. V, n. 60, p. 1172, 1938.

IRIYE, Akira; SAUNIER, Pierre-Yves (Ed.). The Palgrave dictionary of transnational history. Basingstoke: Palgrave Macmillan, 2009.

KELLER, Carlos. Revolución en la agricultura. Santiago: Zig-Zag, 1956.

MATTHEI, Adolfo. Bericht über die Gründung und Organisation der höheren Landwirtschaftlichen Schule zu Osorno. En: MATTHEI, Adolfo. Mitteilungen der höheren Landwirtschaftlichen Schule zu Osorno (Chile). Osorno: Imprenta Wohlwend, 1933. n. 1.

La agricultura en Chile y la política agraria chilena. Santiago: Nascimiento, 1939.

NEUMANN, Alfredo. Aborto contagioso. Agricultura Austral, Osorno, a. IV, n. 37, p. 6-9, 1936a.

vantes, 1946.

Enfermedades del ganado. Osorno: Cer-

Importancia de los registros genealógicos. Agricultura Austral, Osorno, a. IV, n. 47, p. 540-547, 1936b.

Tratamiento de las enfermedades del ganado. Osorno: Cervantes, 1932.

OJEDA-EBERT, Gerardo. Deutsche Einwanderung und Herausbildung der chilenischen Nation: (1846-1920). München: Fink, 1984.

OSORNO debe ser sede de un servicio de ganadería. LA PRENSA, Diario de Osorno. Osorno, 6 de octubre 1935, p. 9.

PERALTA, Gabriel. Historia económica y urbana de Osorno. Osorno: Impresur1991.
POBLETE, Moisés. El problema de la producción agrícola y la política agraria nacional. Santiago: Imprenta Universitaria, 1919.

REVISTA VISUR. Puerto Montt, 1994, p. 5-7.

RINKE, Stefan. Cultura de masas, reforma y nacionalismo en Chile, 1910-1931. Valparaíso: DIBAM, 2002.

. Der letzte freie Kontinent: Deutsche Lateinamerikapolitik im Zeichen transnationaler Beziehungen, 1918-1933. Stuttgart: Heinz, 1996.

RISSE, Thomas. Avances en el estudio de las relaciones internacionales y la política mundial. Foro Internacional, México, DF, v. 39, n. 4, p. 374-403, 1999.

ROBLES, Claudio. Expansión y transformación de la agricultura en una economía exportadora. La transición al capitalismo agrario en Chile (1850-1930). Historia Agraria, Murcia, n. 29, p. 45-80, 2003.

ROMÁN, Jorge; VILLALOBOS, Jovino. Libro del cuarto centenario de Osorno. Osorno: Ilustre Municipalidad de Osorno, 1958.

SANTANA, Roberto. Agricultura chilena en el siglo XX: contextos, actores y espacios agrícolas. Santiago: Dirección de Bibliotecas Archivos y Museos, 2006.

SIEVERS, Hugo. Chile: desarrollo de la medicina veterinaria durante la República (18101970). Santiago: Colegio Médico Veterinario, 1971.

SUBERCASEAUX, Bernardo. Historia de las ideas y la cultura en Chile. Santiago: Editorial Universitaria, 1997. Tomo II.

YOUNG, George F. W. The Germans in Chile: immigration and colonization 1849-1914. New York: Center for Migration Studies, 1974. 


\section{Anexos}

\section{Anex0 1}

Principales publicaciones de Alfredo Neumann Kuschel:

1) Tratamiento de las enfermedades del ganado. Osorno: Cervantes, 1932.

2) Tres problemas que afectan a la producción ganadera. Osorno: Cervantes, 1942.

3) Enfermedades del ganado. Osorno: Cervantes, 1946.

4) Vida y obras de Adolfo Matthei Schwarzenberg. Osorno: [s. n.], 1969.

5) Alexander von Humboldt: su obra y su influencia en América. Osorno: [s. n.], 1971.

6) Don Bernardo Minte Sunkel: homenaje a su memoria. Osorno: [s. n.], 1975.

7) Presencia de la mujer en la inmigración alemana en el sur de Chile. Osorno: [s. n.], 1978.

8) Fundación Adolfo Matthei. Instituto Profesional Agrario. Osorno: [s. n.], 1982. Folleto conmemorativo del cincuentenario.

9) El Pudú: contribución a su estudio etológico. Osorno: Impresur, 1992.

\section{Anexo 2}

Muestra de 20 memorias de titulación en temas pecuarios para optar al título de "Perito Agrícola", ESAO, periodo 19351955. Fuente: Biblioteca Instituto Profesional Agrario Adolfo Matthei de Osorno.

1) KUNTSMANN, Ariberto. Cría y explotación del cerdo en el sur de Chile, 1935.

2) GESCHE, Walter. Das schwarzbunte Niederungsrind in Südchile, 1935.

3) BONGARDT VON TOLL, Hans. Explotación del vacuno, especialmente de los overos negros en el sur de Chile, bajo consideración especial del criadero Los Riscos, 1936.

4) ROIG, John. El durham shorthorn en la zona sur de Chile, 1937.

5) FEHLANDT, Kurt. Fomento del ganado vacuno en Chile, 1938.

6) BORNSCHEUER, Pedro. La fiebre aftosa y sus consecuencias, 1939.

7) WOERNER, Roberto. Conservación de forrajes, 1939.

8) KRUMBACH, Horst. Las familias de overos colorados que se han formado en Chile, 1940.

9) REISY, Othmar. Lechería y sus adelantos, 1940.

10) HOTT, Oswald. Wege zur Verbesserung des südchilenischen Grünlandes, 1942.

11) MARTENS, Gerardo. Die Aufzucht des Jungrindes, 1943.

12) MOHR, Jorge. El proceso de la digestión y aprovechamiento de los alimentos en el cuerpo animal, 1943.

13) WILLER, Rolando. Aprovechamiento de residuos industriales en la alimentación del ganado, 1944.

14) MANRÍQUEZ, Miguel. La fiebre aftosa, 1944.

15) CORONEL, Alejandro. La higienización de la leche, 1944.

16) SCHLICHT, Hugo. Alimentación del ganado lechero, 1945.

17) STROHBACH, Ottomar. Koppelwirtschaft. Neuzeitliche Weidenutzung, 1947.

18) MARECAUX, Juan. Alimentación racional del ganado lechero, 1948.

19) TRAUB, Mario. El shorthorn, overo colorado y overo negro en Chile, 1951.

20) STÜCKRATH, Raúl. Explotación del criadero "El Copihue", 1955. 